\title{
BILAN DE 4 ANNÉES D'ÉTUDE DU COMPORTEMENT REPRODUCTEUR ET DE L'HYBRIDATION CHEZ BARBUS BARBUS (L., 1758) ET BARBUS MERIDIONALIS (RISSO, 1826).
}

\section{P. PONCIN}

Université de Liège, Service d'Ethologie et de Psychologie animale, Laboratoire d'Éthologie des Poissons, 22 quai Van Beneden, 4020 Liège, Belgique.

\section{RÉSUMÉ}

Cet article synthétise les résultats de 4 années d'étude du comportement de reproduction et de l'hybridation chez Barbus barbus (L., 1758) et Barbus meridionalis (Risso, 1826) en aquarium.

On y aborde successivement la description des parades sexuelles, la mise en évidence du comportement d'hybridation, l'étude des rythmes biologiques nycthéméraux et l'influence des stimuli visuels sur les comportements sexuels.

Mots-clés : comportement reproducteur, Barbus barbus, Barbus meridionalis, hybridation, rythmes biologiques.

\section{FOUR YEARS RESEARCH ON THE REPRODUCTIVE BEHAVIOUR AND HYBRIDIZATION OF BARBUS BARBUS AND BARBUS MERIDIONALIS.}

\begin{abstract}
This paper reviews the main results of 4 years studies on the reproductive behaviour of Barbus barbus (L., 1758) and Barbus meridionalis (Risso, 1826) under controlled conditions in aquarium. The reproductive behaviour is described as well as the behavioural mechanisms of hybridization. During the spawning act - sometimes preceded by "forehead swim" in which two fish swim together, at high speed, head against head the female and the few accompanying males rise from the gravel and move their genital papilla in the gravel while releasing ova or sperm (fig. 1 and 2). The reproductive behaviours of Barbus barbus and Barbus meridionalis appear to be similar and hybridizations were observed, showing that no complete behavioural barrier blocks the mating of the two species (fig. 2). The daily rhythms of activity are compared within the two species. The influence of temperature and daylength on the daily rhythms of activity is discussed. In winter conditions, barbels exhibit a night activity rhythm. Spawning occurs during daytime or during the night (fig. 3). The influence of spawning stimuli is analysed using dummies of different sizes and colors (photo I). Barbels seem to prefer white, grey, yellow and brown colors to red or black colors.
\end{abstract}

Key-words : reproductive behaviour, Barbus barbus, Barbus meridionalis, hybridization, biological rhythms. 


\section{INTRODUCTION}

Les espèces européennes du genre Barbus ont fait l'objet de nombreuses études abordant les aspects démographiques, morphologiques, systématiques, biogéographiques,... de leur biologie (BERREBI, 1990). Par contre, peu de travaux sont entièrement consacrés aux comportements agonistique et reproducteur. La première recherche sur ce sujet, réalisée en Angleterre, décrit les stratégies de reproduction, en milieu naturel (HANCOCK et al., 1976). Elle restera unique pendant une dizaine d'années. Il était donc opportun de développer ce type de sujet. Dans un premier temps, profitant de la mise au point de l'élevage contrôlé de l'espèce Barbus barbus (L., 1758) dans les eaux réchauffées du centre de recherche de Tihange (PHILIPPART et al., 1989 ; PONCIN, 1988), GOUGNARD et al. (1987)* commencèrent par décrire le comportement reproducteur du barbeau fluviatile en aquarium, s'attachant à mettre en évidence l'influence du nombre de mâles courtisants sur le succès des pontes. Les résultats encourageants de cette étude nous amenèrent à poursuivre les recherches en nous intéressant à une seconde espèce, Barbus meridionalis (Risso, 1826). En effet, on trouve des hybrides naturels de Barbus barbus et Barbus meridionalis dans le bassin de l'Hérault, dans le sud de la France (BERREBI et al., 1987). En vue de comprendre les modalités d'hybridation des deux espèces et de mieux connaître le barbeau méridional, jusque-là peu étudié en captivité, nous avons développé des recherches en aquarium. C'est ainsi que le comportement d'hybridation et les rythmes biologiques ont été analysés en détail (JEANDARME et al., 1992* ; LEONARD et PONCIN 1993* ; PONCIN et al., $\left.1994^{\star}\right)$. Les travaux ont ensuite été complétés par l'étude de la perception visuelle des deux espèces (LELEU, 1993*). Parallèlement à ces travaux de laboratoire et dans la droite ligne des recherches de HANCOCK et al. (1976), les comportements reproducteur et agonistique du barbeau fluviatile ont aussi fait l'objet d'études remarquables en milieu naturel (BARAS, 1992).

Cet article synthétise les principaux résultats de recherches précitées $\left({ }^{*}\right)$, menées exclusivement en aquarium, au Laboratoire d'Ethologie des Poissons de l'Université de Liège, depuis 1987. II constitue la version complétée et finalisée d'une communication présentée à la table ronde «Barbus Il» et publiée sous forme d'un résumé (PONCIN, 1993).

\section{MATÉRIEL ET MÉTHODES : GÉNÉRALITÉS}

Les barbeaux fluviatiles proviennent du Centre de Recherche (CERER) de Tihange où ils sont élevés en captivité (PHILIPPART et al., 1989). Les barbeaux méridionaux ont été capturés en milieu naturel, dans l'Aubaygue, affluent de la Lergue (bassin de l'Hérault, France).

Les poissons sont maintenus dans des aquariums de 600 à $1000 \mathrm{I}$. Ils sont nourris à satiété au moyen de larves de chironomides surgelées (vers de vase) ou d'asticots vivants. La photopériode et la température sont manipulées, permettant le contrôle des cycles de reproduction, selon la méthode décrite chez Barbus barbus par PONCIN et CASTELLI (1990). Un courant d'eau est créé dans chaque aquarium $( \pm 21 \mathrm{~cm} / \mathrm{sec})$ au moyen d'une pompe. Chacun est muni d'un abri en PVC suspendu à $20 \mathrm{~cm}$ du fond qui est tapissé de graviers ( $\pm 5 \mathrm{~mm}$ de diamètre), indispensables pour l'expression des comportements reproducteurs.

Les observations comportementales sont enregistrées à l'aide d'une caméra et d'un magnétoscope, commandés à partir d'un local adjacent au laboratoire. De nuit, des spots infrarouges [longueur d'onde supérieure à $790 \mathrm{~nm}$ pour ne pas déranger les poissons (BEACH, 1978)] et une caméra de surveillance permettent les observations.

Afin de stimuler et de synchroniser l'activité reproductrice des poissons et d'induire la ponte, on a parfois recours à une technique d'injection d'extraits hypophysaires de carpe suivant la méthode appliquée par KOURIL et al. (1988) chez Barbus barbus. L'évolution des comportements au cours du temps a été détaillée par JEANDARME et al. (1992). Elle est comparable chez les deux espèces de Barbus. Les comportements reproducteurs apparaissent après environ 9 heures à $23^{\circ} \mathrm{C}$. 


\section{DESCRIPTION DU COMPORTEMENT REPRODUCTEUR}

Les comportements reproducteurs de $B$. barbus et $B$. meridionalis sont fort semblables. Ils correspondent à la description détaillée réalisée par GOUGNARD et al. (1987) chez $B$. barbus. Lors d'une séquence comportementale classique, la femelle, accompagnée de plusieurs mâles (1 à 6-7) nage rapidement dans tout l'aquarium, quittant fréquemment l'abri qui lui sert de refuge. Mâles et femelles effectuent souvent des nages de front, qui consistent en une nage rapide effectuée par deux individus placés côte à côte, la tête de l'un se maintenant au niveau de l'opercule de l'autre. Ce n'est qu'un ou deux jours avant une première tentative de frai que l'on enregistre les premières nages de front. Elles s'observent aussi bien entre deux mâles qu'entre un mâle et une femelle (nages de front mixtes), et ne sont pas essentielles au bon déroulement d'une séquence comportementale. Elles jouent surtout un rôle de synchronisation. Elles s'observent plus rarement chez $B$. meridionalis. Ensuite, la femelle, toujours accompagnée des mâles, se dirige vers le fond de l'aquarium pour y effectuer une "tentative de ponte". Redressée, nageoires déployées, bouche projetée vers l'avant et opercules écartés, elle agite sa papille génitale dans le gravier. Avec un comportement comparable, les mâles, accolés aux flancs de la femelle, émettent leur sperme (figures 1 et 2). Cette séquence peut se répéter jusqu'à 280 fois pendant une période de 10-11 heures de ponte (PONCIN et al., 1994). En aquarium, chaque fois que la femelle agite sa papille génitale dans le gravier, on dénombre environ une cinquantaine d'oeufs émis dont la survie est grossièrement estimée à $50 \%$ (LELEU, 1993).

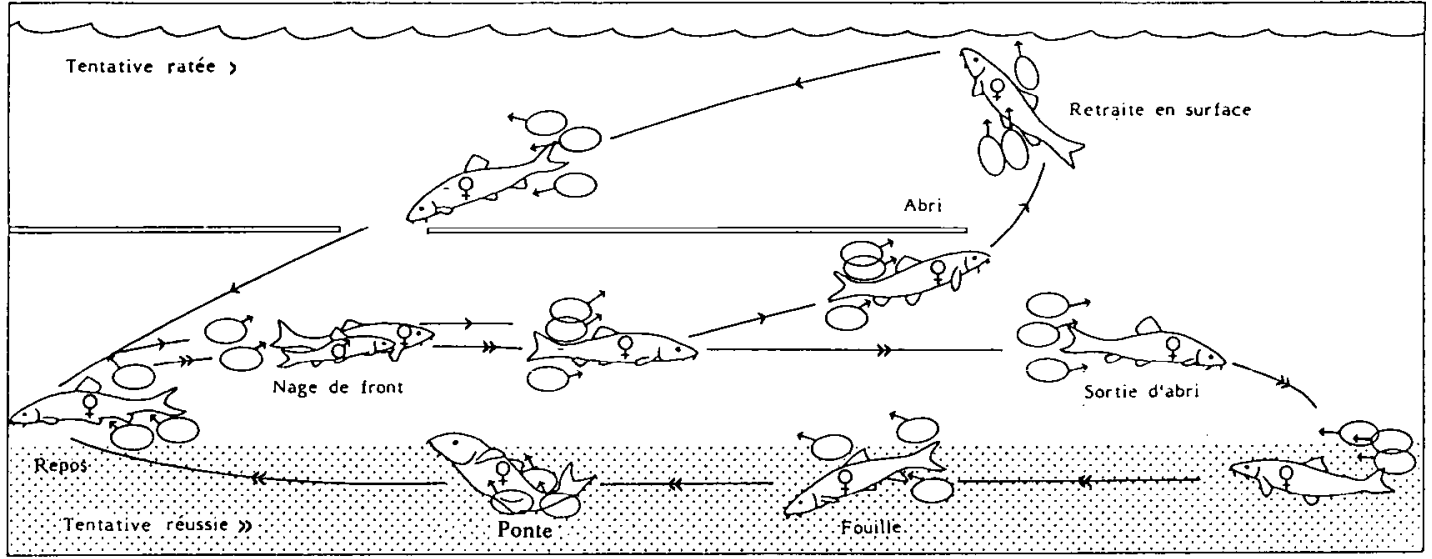

Figure 1 : Séquences comportementales observées chez Barbus barbus ( 1 femelle $Q$ et 3 mâles $\sigma^{\prime}$ ), aboutissant à une ponte (tentative réussie) ou à une retraite en surface (tentative ratée). Adapté d'après GOUGNARD et al., 1987.

Figure 1 : Behavioural sequences observed in Barbus barbus (1 female $Q$ and 3 males $O^{7}$ ), which end with spawning (successful attempt) or escape to surface (failed attempt). Modified from GOUGNARD et al., 1987.

L'évolution du comportement au cours des cycles de reproduction [une ponte tous les 8 jours en captivité, (PONCIN, 1988)] ainsi que l'influence du nombre de mâles courtisants ( 3 ou 6 pour une femelle) ont aussi été décrites en détail par GOUGNARD et al. (1987) chez B. barbus. Entre deux pontes successives, les barbeaux présentent une activité agonistique. Le nombre de mâles courtisants affecte la séquence et la distribution de certains comportements. Des données semblables n'ont pas encore été recueillies chez Barbus meridionalis. 


\section{ÉTUDE COMPORTEMENTALE DE L'HYBRIDATION}

Des lots expérimentaux conspécifiques et hétérospécifiques de barbeaux fluviatiles et méridionaux ont été constitués en vue d'étudier les possibilités d'hybridation des deux espèces (JEANDARME et al., 1992 ; PONCIN et al., 1994, sous presse). L'activité reproductrice des individus est induite par l'injection d'extraits hypophysaires de carpes. Dans ces conditions, on observe de nombreuses hybridations (figure 2) et les oeufs produits dans les différentes combinaisons montrent qu'il n'existe pas de barrière comportementale à l'hybridation des deux espèces. Des comportements d'hybridation ont même été observés récemment en l'absence de stimulation hormonale. Ces résultats complètent les données obtenues par reproduction artificielle (PHILIPPART et BERREBI, 1990) où des hybrides femelles féconds ont été obtenus (les mâles étaient stériles). L'absence de découverte d'hybride de première génération dans le sud de la France indique que le croisement des deux espèces est actuellement inexistant. La présence d'une "zone hybride» (BERREBI et al., 1987) de quelques kilomètres de long constitue une barrière empêchant les deux espèces de se rencontrer, mais permettant éventuellement leur croisement avec des individus hybrides.
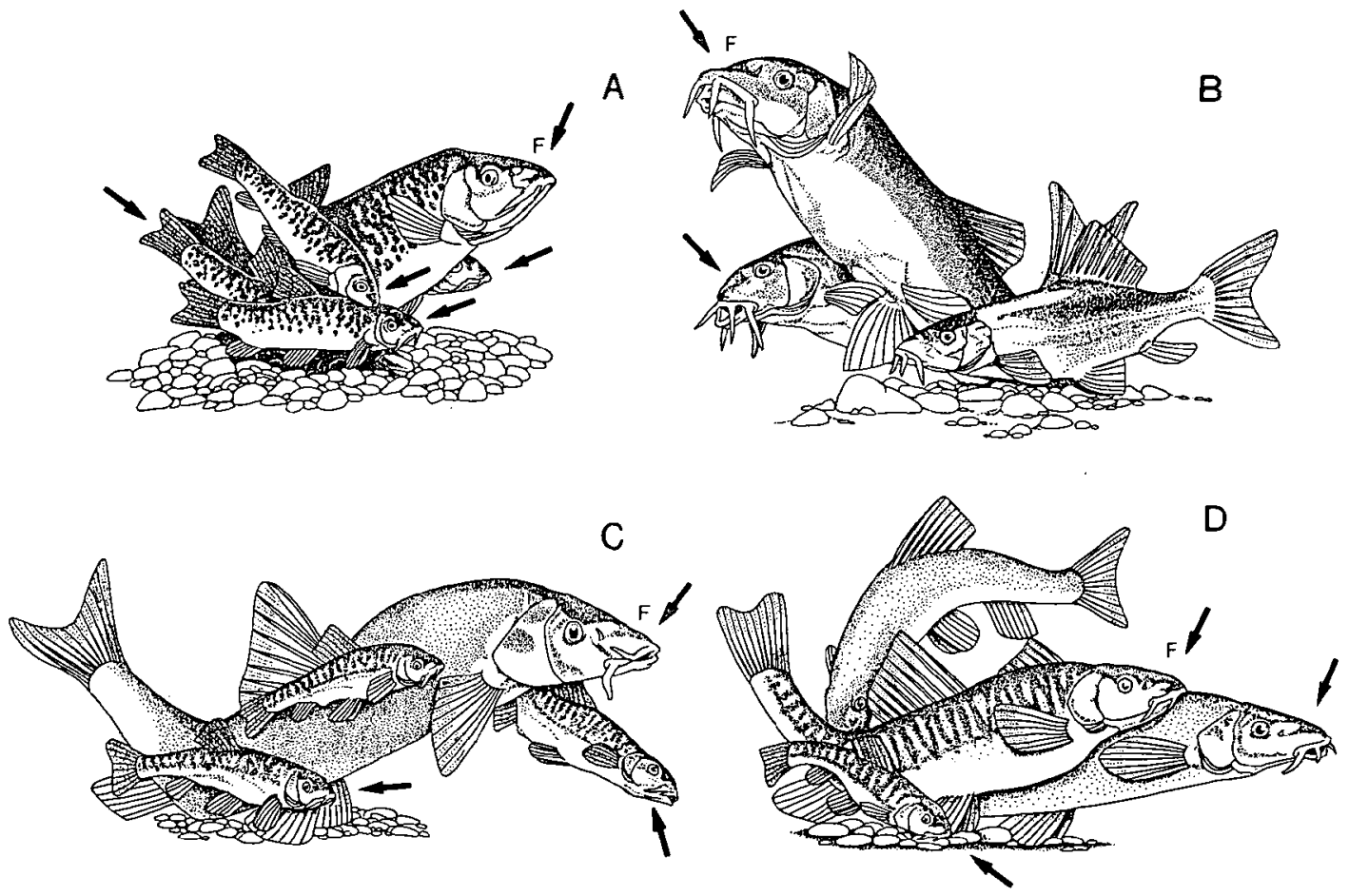

Figure 2 : Comportement de ponte d'une femelle Barbus barbus (B et $C$ ) ou Barbus meridionalis (A et $D$ ) accompagné de mâles conspécifiques (A et $B)$, hétérospécifiques (C) ou hétéro et conspécifiques (D). Les flèches indiquent les poissons qui libèrent du sperme ou des ovules. $A$ : d'après JEANDARME et al., 1992 ; B : d'après PONCIN et al., 1994. F = femelle.

Figure 2 : Spawning behaviour of a female Barbus barbus ( $B$ and $C$ ) or Barbus meridionalis (A and $D$ ) and conspecific (A and B), heterospecific (C) or hetero and conspecific (D) males. Arrows indicate fish releasing sperm or ova. A : from JEANDARME et al., 1992 ; B : from PONCIN et al., 1994. $F=$ female. 
D'autre part, on peut aussi supposer l'existence de barrières écologiques (situation spatiale des frayères, exigences en oxygène, ...) et phénologiques (dates de pontes légèrement différentes) qui tendraient à isoler les deux espèces de barbeaux.

\section{COMPARAISON DES RYTHMES BIOLOGIQUES NYCTHÉMÉRAUX}

Les rythmes nycthéméraux des deux espèces ont été décrits sous différentes conditions de photopériode et de température (LEONARD et PONCIN, 1993), à savoir :

— conditions "post-hivernales»: $8 \mathrm{~L}: 16 \mathrm{~N} ; 10-12^{\circ} \mathrm{C}$.

— conditions «printanières»: $12 \mathrm{~L}: 12 \mathrm{~N} ; 14-15^{\circ} \mathrm{C}$.

— conditions "estivales": $16 \mathrm{~L}: 8 \mathrm{~N} ; 19-20^{\circ} \mathrm{C}$.

Les deux espèces sont essentiellement nocturnes en conditions «post-hivernales». II apparaît toutefois que les barbeaux méridionaux sont globalement plus actifs et sortent plus souvent de leur abri que les fluviatiles. Chez les deux espèces, l'activité s'accroît lorsqu'on augmente la température et la photopériode. On voit alors apparaître une composante diurne. En conditions estivales, l'activité hors ou sous abris est proche de $100 \%$ chez les deux espèces, mais on observe une nette différence dans l'occupation de l'abri ( $B$. meridionalis sort plus souvent). Les différences observées reflètent probablement la disparité des habitats des deux espèces (rivière large chez $B$. barbus ; petits ruisseaux chez $B$. meridionalis). D'une manière générale, en ce qui concerne $B$. barbus en captivité, l'évolution de l'activité des poissons en fonction de la température et de la photopériode corrobore les informations recueillies par BARAS (1992) en milieu naturel, où il apparaît que l'activité journalière des barbeaux est liée à l'augmentation printanière de la température et qu'ils exhibent un patron journalier d'activité bimodal en été.

L'observation des rythmes nycthéméraux de ponte a permis de mettre en évidence qu'en l'absence de variation journalière de la température, la ponte des barbeaux peut se réaliser de jour comme de nuit (figure 3). Dans le premier cas, on note une véritable inversion du cycle d'activité, qui de nocturne devient diurne. Ces observations sont aussi en parfait accord avec celles réalisées en rivière par BARAS (1992).

Enfin, des recherches préliminaires sur l'influence d'une diminution d'intensité lumineuse (980-1050 luxs $->0,8$ lux ou 980-1050 luxs $\rightarrow<0,8$ lux) sur les comportements laissent entrevoir un effet de cette dernière sur la fréquence de frai. Le passage à une luminosité $<0,8$ lux induirait une diminution de la fréquence de ponte des femelles.

\section{ÉTUDE DE LA PERCEPTION VISUELLE}

Les capacités de perception visuelle des deux espèces de barbeaux, caractérisées au moyen de coupes histologiques semi fines de la rétine, sont similaires (LELEU, 1993 ; COMPERE et al., en préparation). Les barbeaux possèdent une vision chromatique qui, du fait de la faible densité en récepteurs (cônes), n'est certainement effective qu'en lumière vive.

Il semble que l'agitation de la femelle constitue le principal stimulus incitant les mâles à participer à la ponte. Cette hypothèse, qui s'appuie sur des résultats obtenus sur le saumon par SATOU et al. (1991), a été testée au moyen de leurres de formes et de couleurs différentes (plaques rectangulaires en polycarbonates ou moulages de barbeaux en résine polyester), présentés en mouvement, aux poissons (LELEU, 1993). Les mâles Barbus barbus ou Barbus meridionalis réagissent positivement au leurre allant juqu'à s'en approcher et se placer parallèlement à celui-ci (photo I). Des comportements comme "l'attention attirée par le leurre", "la sortie d'abri», "la poursuite du leurre» ou «l'approche» peuvent faire l'objet d'une quantification. Dans ce cas, il apparaît que la taille du leurre $(25 \times 5 \mathrm{~cm}$ ou $40 \times 10 \mathrm{~cm}$ pour les plaques rectangulaires -25 et $40 \mathrm{~cm}$ pour les imitations de barbeaux) ne semble pas influencer significativement le comportement des mâles. Par contre, la coloration du leurre détermine l'intensité de la réponse comportementale. Les barbeaux préfèrent le blanc, le gris, le jaune et le brun plutôt que le rouge ou le noir. Ce résultat n'est pas étonnant dans la mesure où les quatre couleurs préférées se retrouvent dans les patrons de coloration des deux espèces étudiées. 

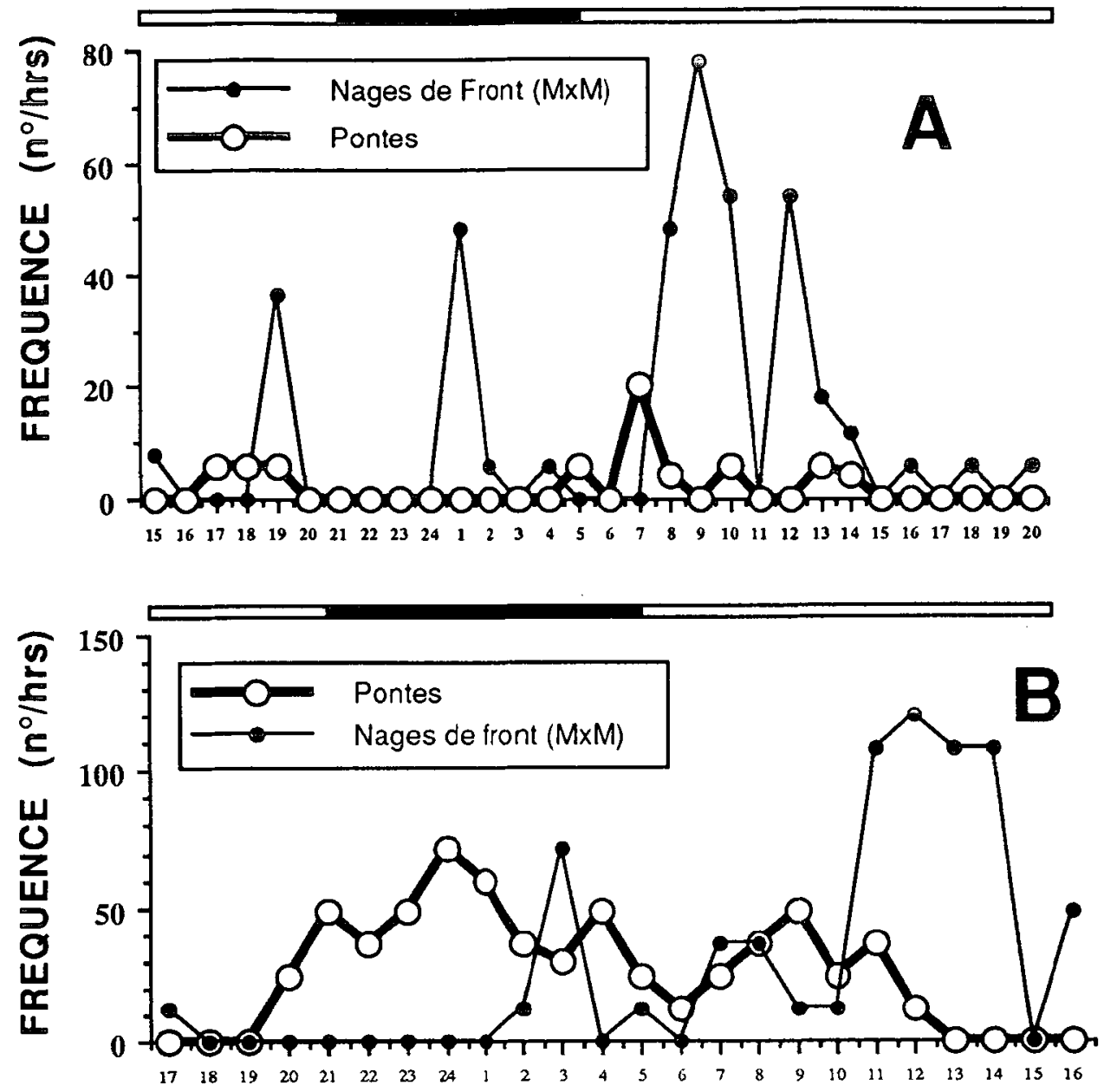

HEURES

Figure 3 : Pourcentage journalier de pontes et de nages de front (entre mâles) dans deux groupes de barbeaux ( 1 femelle $\times 4$ mâles). A : ponte durant le jour ; $B$ : ponte qui débute de nuit et se poursuit le jour.

Figure 3 : Daily frequency of spawning acts and forehead swims between males in 2 groups of barbels ( 1 female $\times 4$ males). A : spawning during the day; $B$ : spawning during the night and continuing during the day.

\section{CONCLUSION}

Les recherches présentées brièvement dans cet article développent un aspect peu connu de la biologie des Barbus : le comportement reproducteur et les rythmes biologiques en conditions contrôlées. Elles complètent les travaux menés sur le terrain (par radiopistage, BARAS, 1992, par exemple), en apportant des éléments de discussion nouveaux. Elles apportent aussi des éléments de discussion plus générale sur la plasticité du comportement des Barbus ou sur les problèmes de spéciation et d'évolution des espèces de poissons. Ce thème de recherche trouve son application dans l'étude des modalités d'hybridation des poissons, et plus particulièrement des barbeaux, en milieu naturel, où une 


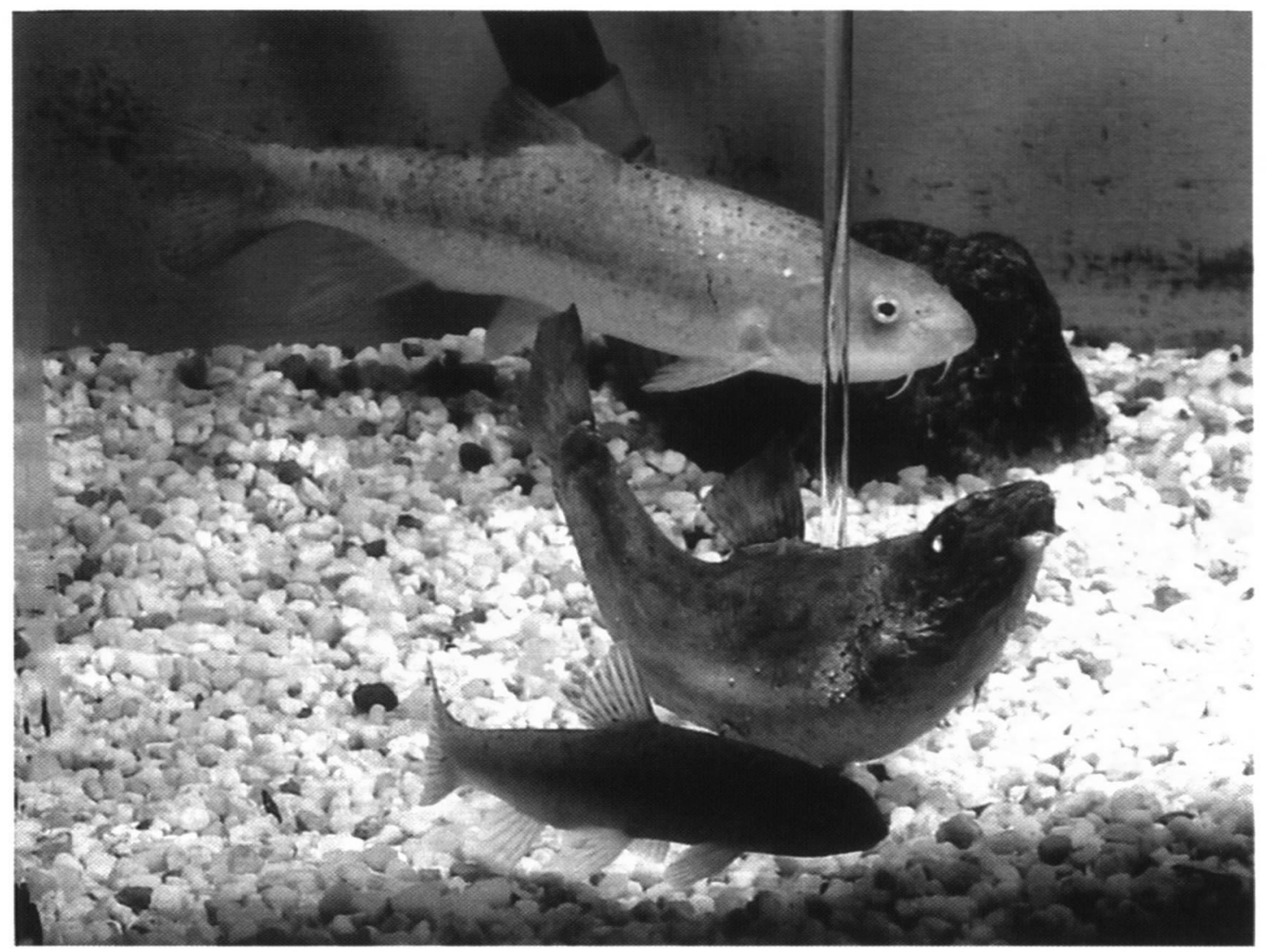

Photo I : Un mâle barbeau (Barbus meridionalis) courtisant un leurre imitant une femelle en posture de ponte. Une femelle méridionale est aussi visible en arrière-plan.

Photo I: A male barbel (Barbus meridionalis) courting a dummy mimicking a spawning female. A female meridionalis stays in the background.

approche éthologique des mécanismes s'avère souvent très difficile. L'étude du comportement en aquarium vient alors en complément d'autres travaux, sur la génétique, la physiologie, la morphologie ou la dynamique des populations.

\section{REMERCIEMENTS}

Ces études ont été menées avec l'appui financier du Fonds National de la Recherche Scientifique (FNRS, crédit $n^{\circ}$ 9.4584.91) et de la Loterie Nationale, ainsi que de la Commission Piscicole Provinciale du Fonds Piscicole de Liège. Qu'ils en soient remerciés.

L'auteur remercie aussi les Drs J.C. RUWET, J.C. PHILIPPART et P. BERREBI pour leur soutien, Ph. HUMPERS, S. HOUBART, J.M. BERTHOLET, R. MARECHAL pour leur aide technique, ainsi que les étudiants thésards ou stagiaires qui ont réalisé ou participé aux recherches : I. GOUGNARD, J. JEANDARME, L. LEONARD et C. LELEU. 


\section{BIBLIOGRAPHIE}

BARAS E., 1992. Etude des stratégies d'occupation du temps et de l'espace chez le barbeau fluviatile, Barbus barbus (L.). Cah. Ethol., 12 (2-3), 125-442.

BEACH M.H., 1978. The use of infra-red light and closed circuit TV to validate records from automatic fish counters. J. Fish Biol., 13, 639-644.

BERREBI P., 1990. The "Barbus" model (Cyprinidae). A focus of multidisciplinary European interest. Aquat. Living Resour., 3 (4), éditorial.

BERREB| P., LE BRUN N., RENAUD F., LAMBERT A., 1987. Hybridation inter-spécifique de deux Cyprinidae (genre Barbus). Conséquence sur la spécificité parasitaire de D. gracile (Monogenea). In Actes du Colloque National du CNRS, «Biologie des Populations» (I.A.S.B.S.E., Université Claude Bernard, ed.). Lyon, France, 699 p.

GOUGNARD I., PONCIN P., RUWET J.C., PHILIPPART J.C., 1987. Description et analyse du comportement de reproduction du barbeau Barbus barbus (L.) en aquarium. Influence du nombre de mâles courtisants sur les comportements observés. Cah. Ethol. appl., 7 (3), 293-302.

HANCOCK R.S., JONES J.W., SHAW R., 1976. A preliminary report on the spawning behaviour and nature of the sexual selection in the barbel, Barbus barbus (L.). J. Fish Biol., $9(1), 21-28$.

JEANDARME J., PONCIN P., BERREBI P., 1992. Etude préliminaire du comportement d'hybridation de Barbus barbus ( $L$.) et Barbus meridionalis (Risso) en aquarium. Cah. Ethol., 12 (4), 519-528.

KOURIL J., FILLA V., SANDERA K., BARTH T., FLEGEL M., 1988. Hormonally induced stripping of the female barbel (Barbus barbus L.) using carp pituitary and LH-RH analog. Bul. VURH Vodnany, 3, 18-25.

LELEU C., 1993. Etude de la perception visuelle chez 2 espèces du genre Barbus : Barbus barbus (L.) et Barbus meridionalis (Risso) en relation avec l'hybridation. Mémoire de Licence, Université de Liège, $53 p+$ annexe.

LEONARD L., PONCIN P., 1993. Influence de la température et de la durée d'éclairement sur les rythmes nycthéméraux d'activité chez Barbus barbus et Barbus meridionalis. Cah. Ethol., 13 (2), 181-182.

PHILIPPART J.C., BERREBI P., 1990. Experimental hybridization of Barbus barbus and Barbus meridionalis: physiological, morphological, and genetic aspects. Aquat. Living Resour., 3, 325-332.

PHILIPPART J.C., MELARD C., PONCIN P., 1989. Intensive culture of the common barbel, Barbus barbus (L.) for restocking. In Aquaculture - a biotechnology in progress ( $N$. De Pauw, E. Jaspers, H. Ackefors, N. Wilkins, eds), 483-491, European Aquaculture Society, Bredene, Belgium.

PONCIN P., 1988. Le contrôle environnemental et hormonal de la reproduction en captivité du barbeau et du chevaine. Cah. Ethol. appl., 8 (2), 173-336.

PONCIN P., 1993. Le comportement reproducteur et l'hybridation chez Barbus barbus et Barbus meridionalis en aquarium. Brève revue. Cah. Ethol., 13 (2), 147-150.

PONCIN P., CASTELLI M., 1990. Manipulation photopériodique des saisons de reproduction chez le barbeau (Barbus barbus). Bilan de deux années d'application des techniques. Cah. Ethol. appl., 10 (3-4), 447-450.

PONCIN P., JEANDARME J., BERREBI P., 1994. A behavioural study of hybridization between Barbus barbus and Barbus meridionalis. J. Fish Biol., 45, 447-451.

SATOU M., TAKEUCHI H.A., NISHII J., TANABE M., KITAMURA S., KUDO Y., OKUMOTO N., 1991. Inter-sexual vibrational communication during spawning behaviour in the himé salmon (landlocked red salmon, Oncorhynchus nerka). In Reproductive Physiology of Fish (Scott A.P., Sumpter J.P., Kime D.E. \& Rolfe M.S., eds), 185-187, FishSymp 91, Sheffield, U.K. 